\title{
Screening Relevance of Sessile Serrated Polyps
}

\author{
Charles J. Kahi \\ Department of Medicine, Indiana University School of Medicine, Richard L. Roudebush VA Medical Center, Indianapolis, IN, USA
}

Conventional adenomas have historically been considered to be the only screening-relevant colorectal cancer (CRC) precursor lesion. The prevailing paradigm was that most CRCs arise along the chromosomal instability pathway, where adenomas accumulate incremental genetic alterations over time, leading eventually to malignancy. However, it is now recognized that this "conventional" pathway accounts for only about two-thirds of CRCs. The serrated pathway is responsible for most of the remainder, and is a disproportionate contributor to postcolonoscopy CRC. Hallmarks of the serrated pathway are mutations in the BRAF gene, high levels of methylation of promoter CpG islands, and the sessile serrated polyp (SSP). Accumulating evidence shows that SSPs can be considered adenoma-equivalent from the standpoint of CRC screening. SSPs have a higher prevalence than previously thought, and appear to have a relatively long dwell time similar to that of conventional adenomas. In addition, SSPs, whether sporadic or as part of the serrated polyposis syndrome, are associated with increased risk of synchronous and metachronous neoplasia. These features collectively support that SSPs are highly relevant to CRC prevention. Clin Endosc 2019;52:235-238

Key Words: Colorectal neoplasms; Screening; Colonoscopy

\section{INTRODUCTION}

Colorectal cancer (CRC) is amenable to primary prevention, which means that incidence and mortality can be reduced via screening and removal of precursor lesions before cancer occurs. Successful CRC screening fundamentally depends on the ability to detect and resect precursor lesions with relatively long dwell time. This paradigm has long been established for conventional adenomas, but the more recent emergence of serrated lesions in the CRC field has led to questions about its applicability to sessile serrated polyp (SSP). There are presently no prospective studies showing a measurable independent impact of SSP removal on CRC outcomes. This may be largely due to the relatively recent recognition of the importance of SSP in the CRC prevention field, compared to the very ex-

Received: June 27, 2018 Accepted: July 10, 2018

Correspondence: Charles J. Kahi

Department of Medicine, Indiana University School of Medicine, Richard L. Roudebush VA Medical Center, 1481 West 10th street, Indianapolis, IN 46202, USA

Tel: +1-317-988-3682, Fax: +1-317-988-5313, E-mail: ckahi2@iu.edu ORCID: https://orcid.org/0000-0003-0907-8835

cc This is an Open Access article distributed under the terms of the Creative Commons Attribution Non-Commercial License (http://creativecommons.org/ licenses/by-nc/3.0) which permits unrestricted non-commercial use, distribution, and reproduction in any medium, provided the original work is properly cited. tensively studied conventional adenomas. Nevertheless, an extensive and growing literature provides several converging lines of evidence suggesting that SSP have a central role in CRC prevention. Recent studies describe the distinct biologic features of the serrated pathway to colorectal neoplasia, and reveal that SSP have high prevalence, a relatively long dwell time, and are associated with increased risk of both synchronous and metachronous colorectal neoplasia. A review of this literature is presented in the following sections.

\section{THE SERRATED PATHWAY}

Serrated polyps are distinct from conventional adenomas and are characterized histologically by a saw-tooth appearance of the crypt epithelium. ${ }^{1}$ This class of lesions includes hyperplastic polyps, SSP, and traditional serrated adenomas (TSA). SSP and TSA are both considered premalignant lesions; however, TSA are very rare lesions and are less well-understood than SSP. Distortion of crypt growth pattern leading to abnormal-appearing crypt bases is characteristic of SSP. An activating mutation of the BRAF oncogene appears to be a fundamental step in the SSP-to-CRC serrated neoplasia pathway, and a characteristic feature is abnormal hypermeth- 
ylation of $\mathrm{CpG}$ islands in gene promoter regions, termed $\mathrm{CpG}$ island-methylator phenotype (CIMP). This hypermethylation can be extensive and lead to epigenetic silencing of genes, such as the mutation mismatch repair gene MLH1 (also implicated in Lynch syndrome). This event leads to acceleration of development of cytological dysplasia (via the intermediate SSP with cytological dysplasia), and progression to CRC with microsatellite instability (MSI). Most CRCs with MSI and CIMP molecular signatures originate from the serrated pathway, with the SSP as the main precursor lesion. ${ }^{2}$

Additional evidence supporting the existence of a separate serrated pathway to CRC includes the observation that SSP may have risk factors distinct from those typically associated with adenomas. For example, calcium and vitamin D supplementation are associated with increased risk of SSP, ${ }^{3}$ while obesity, folate intake, fiber and fat intake are not.

\section{NATURAL HISTORY OF SSP}

The natural history of SSP and rate of progression to CRC are not as clear compared to conventional adenomas and the adenoma-carcinoma sequence. However, the available evidence suggests comparable progression rates, with SSPs generally following an indolent or slowly progressive course, until a critical event (notably, MLH1 silencing) which accelerates the progression to dysplasia and cancer.

A pathological series ${ }^{5}$ of 2,319 patients with 2,416 SSPs reported that the median age of patients increased as the histologic grade of the lesion worsened, from 61 years for SSP without dysplasia, to 76 years for CRC. A recent cross-sectional analysis ${ }^{6}$ of 137 SSPs with dysplasia or cancer showed that older age, female gender, proximal location, CIMP, and lack of aberrant p53 were all associated with loss of MLH1 expression. The age of patients with dysplastic SSP was similar to those with cancer (about 76 years), and together, 17 years older than a historical cohort of patients with SSP without dysplasia.

Collectively, these data show that SSP without dysplasia generally have a long dwell time of at least 15 years, similar to conventional adenomas. This is a critically important observation, because uniformly rapid progression of serrated neoplasms would have threatened current CRC prevention paradigms which rely on relatively long latency of CRC and its precursor lesions.

\section{PREVALENCE}

Until recently, there has been uncertainty regarding the true prevalence of SSP. Older studies addressing this question were limited because they reported aggregate data for endoscopists with wide ranges in individual detection rates, and because there is significant inter-observer variability among pathologists in the differentiation of serrated lesion subtypes. The true prevalence of SSP is important to define, because a rare lesion would not be a reasonable target to screen for. Recent colonoscopy studies ${ }^{7,8}$ involving endoscopists with high-level detection rates reported SSP prevalence of $8 \%$ to $9 \%(0.4 \%$ to $0.6 \%$ for SSP with cytological dysplasia). These studies show that SSP have higher prevalence than previously thought, adding further support to their screening relevance.

\section{SERRATED NEOPLASIA AND POSTCOLONOSCOPY CRC}

There is significant overlap in the molecular signatures of postcolonoscopy CRC (PCCRC) and the serrated pathway. Compared to sporadic CRC, PCCRC is more likely to demonstrate MSI and be associated with CIMP. In addition, PCCRC is more likely to be located in the proximal colon, ${ }^{9-11}$ the area of predilection for SSP.

The majority of PCCRC are due to operator-dependent factors, and this is reflected in its inverse association with adenoma detection rate (ADR). The issues surrounding variable adenoma detection and its impact on the effectiveness of colonoscopy may also apply to serrated polyps, although there is currently no evidence that the variability in proximal serrated polyp detection is independently associated with PCCRC risk. Several studies have shown that the variability in detection of proximal serrated polyps exceeds that of adenomas among the same groups of endoscopists, and can range up to 18- fold (compared to 3- to 4- fold for the ADR). ${ }^{12-14}$ SSPs have subtle morphology which renders them more likely to be overlooked by operators not familiar with their endoscopic characteristics. Longer withdrawal time is significantly associated with improved proximal serrated polyp detection. An analysis ${ }^{15}$ of nearly 8,000 colonoscopies in the New Hampshire Colonoscopy Registry found that the detection of serrated polyps proximal to the sigmoid colon increased with each minute of withdrawal time above 6 minutes. Studies have generally shown a strong correlation between adenoma and serrated polyp detection, ${ }^{13}$ which is plausible because endoscopists who have meticulous examination technique would be expected to be proficient at detecting polyps of all types.

Their endoscopic features also render serrated polyps prone to incomplete resection, as shown in the Complete Adenoma Resection study. ${ }^{16}$ However, more recent studies show that dedicated endoscopic mucosal resection methods, with submuco- 
sal injection with dye to highlight lesion borders, are associated with effective and complete resection of serrated polyps. ${ }^{17,18}$

\section{ASSOCIATION WITH SYNCHRONOUS NEOPLASIA}

Several lines of evidence show that serrated polyps not only possess intrinsic risk of progression to CRC, but also alter the colorectal milieu in a manner which promotes neoplastic processes. A systematic review and meta-analysis ${ }^{19}$ of 9 studies with 34,000 subjects reported that individuals with serrated polyps had a 2 -fold increased odds of advanced neoplasia; proximal location and large size were associated with even higher odds. The most extreme manifestation of serrated neoplasia is the serrated polyposis syndrome (SPS), which is defined as the presence of at least 5 serrated polyps proximal to the sigmoid colon with 2 or more measuring at least 10 $\mathrm{mm}$ in diameter, any number of serrated polyps proximal to the sigmoid colon in a person with a first-degree relative with SPS, or $>20$ serrated polyps of any size distributed throughout the colon. ${ }^{1}$ SPS is the most common polyposis syndrome, with reported prevalence rates ranging from 1 in 238 to 1 in 111 in colonoscopy and fecal test-based screening programs, respectively ${ }^{20,21}$ SPS is associated with increased risk of CRC, warranting close endoscopic surveillance of patients with SPS. ${ }^{22}$ Recent reports, ${ }^{23}$ however, suggest that the risk of CRC in SPS is highest at the time of diagnosis: in a Spanish study of 296 SPS patients, CRC was found in 47 patients at mean age of 54 years, and only 4 cases were detected during surveillance (5year incidence of 1.9\%).

\section{ASSOCIATION WITH METACHRONOUS NEOPLASIA}

Convincing evidence shows that SSP are associated with risk of metachronous neoplasia. A recent study ${ }^{24}$ reported the long-term follow-up of 13,000 individuals in the Norwegian flexible sigmoidoscopy screening trial (NORCCAP), where patients with serrated polyps were re-invited for colonoscopy over a decade after initial enrollment. Compared with controls without polyps, the hazard ratio for CRC was 2.5 for patients with large serrated polyps, comparable to those who had an advanced adenoma (2.0). Interestingly, there were 23 large serrated polyps found at screening were left in situ for a median of 11 years, and none had progressed to CRC; this observation is further evidence that SSP exert a pro-neoplastic influence on the colorectal milieu, independent of their intrinsic risk of progression. A large population-based study ${ }^{25}$ from Denmark assessed the risk of CRC associated with SSP. Among 272,342 individuals who had received colonoscopy between 1977 and 2009, there were 2,045 CRC cases and 8,105 age- and sexmatched controls without CRC. The investigators identified the first colorectal polyp(s) that had been resected or biopsied on the initial or subsequent colonoscopy, and polyps that were initially interpreted as hyperplastic were re-reviewed by expert pathologists. Patients with SSP are at increased risk for CRC, to a degree at least equivalent to that of patients with conventional adenomas (Table 1$)^{25}$

A recently published analysis ${ }^{26}$ of the New Hampshire population-based colonoscopy registry showed that patients with large serrated polyps or SSP at baseline had higher risk of large serrated polyps at follow-up (odds ratio, 9.7 to 14.3), whereas patients with high-risk adenoma (HRA) plus SSP had risk of metachronous HRA which was 4 times higher than those with baseline HRA alone.

\section{CONCLUSIONS}

SSPs contribute significantly to the burden of CRC, and recognition of their importance has direct implications for $\mathrm{CRC}$ screening paradigms. The existence of a distinct biologic

Table 1. Odds of Colorectal Cancer according to Baseline Polyp Type ${ }^{25}$

\begin{tabular}{lcc}
\hline & Adjusted OR (95\% CI) & Estimated 10-year risk \\
\hline SSP with synchronous conventional adenomas & $2.66(1.70-4.16)$ & $2.47 \%$ \\
SSP without synchronous conventional adenomas & $3.40(2.35-4.91)$ & $3.16 \%$ \\
SSP with cytologic dysplasia & $4.76(2.59-8.73)$ & $4.43 \%$ \\
SSP without cytologic dysplasia & $2.75(1.99-3.80)$ & $2.56 \%$ \\
Conventional adenomas without SSP & $2.50(2.24-2.80)$ & $2.33 \%$ \\
TSA overall & $4.84(2.36-9.93)$ & $4.50 \%$ \\
Hyperplastic polyps only & $1.30(0.96-1.77)$ & $1.21 \%$ \\
\hline
\end{tabular}

CI, confidence interval; OR, odds ratio; SSP, sessile serrated polyp; TSA, traditional serrated adenomas. 
pathway, high prevalence, relatively long dwell time, relation to PCCRC, and association with synchronous and metachronous neoplasia render SSPs highly screening-relevant. Continued efforts to ensure that endoscopists achieve high levels of detection and complete resection are critical for effective CRC prevention at colonoscopy.

\section{Conflicts of Interest}

The author has no financial conflicts of interest.

\section{REFERENCES}

1. Snover DC, Ahnen DJ, Burt RW, Odze RD. Serrated polyps of the colon and rectum and serrated ("hyperplastic") polyposis. In: Bozman FT, Carneiro F, Hruban RH, Theise N, eds. WHO classification of tumours of the digestive system. 4th ed. Berlin: Spinger-Verlag; 2010. p. 120-126.

2. Snover DC. Update on the serrated pathway to colorectal carcinoma. Hum Pathol 2011;42:1-10.

3. Crockett SD, Barry EL, Mott LA, et al. Calcium and vitamin D supplementation and increased risk of serrated polyps: results from a randomised clinical trial. Gut 2018 Mar 1 [Epub]. https://doi.org/10.1136/ gutjnl-2017-315242.

4. Davenport JR, Su T, Zhao Z, et al. Modifiable lifestyle factors associated with risk of sessile serrated polyps, conventional adenomas and hyperplastic polyps. Gut 2018;67:456-465.

5. Lash RH, Genta RM, Schuler CM. Sessile serrated adenomas: prevalence of dysplasia and carcinoma in 2139 patients. J Clin Pathol 2010;63:681686.

6. Bettington M, Walker N, Rosty C, et al. Clinicopathological and molecular features of sessile serrated adenomas with dysplasia or carcinoma. Gut 2017;66:97-106.

7. Abdeljawad K, Vemulapalli KC, Kahi CJ, Cummings OW, Snover DC, Rex DK. Sessile serrated polyp prevalence determined by a colonoscopist with a high lesion detection rate and an experienced pathologist. Gastrointest Endosc 2015;81:517-524.

8. IJspeert JE, de Wit K, van der Vlugt M, Bastiaansen BA, Fockens P, Dekker E. Prevalence, distribution and risk of sessile serrated adenomas/ polyps at a center with a high adenoma detection rate and experienced pathologists. Endoscopy 2016;48:740-746.

9. Arain MA, Sawhney M, Sheikh S, et al. CIMP status of interval colon cancers: another piece to the puzzle. Am J Gastroenterol 2010;105:11891195.

10. Sawhney MS, Farrar WD, Gudiseva S, et al. Microsatellite instability in interval colon cancers. Gastroenterology 2006;131:1700-1705.
11. Nishihara R, Wu K, Lochhead P, et al. Long-term colorectal-cancer incidence and mortality after lower endoscopy. N Engl J Med 2013;369:1095-1105.

12. Hetzel JT, Huang CS, Coukos JA, et al. Variation in the detection of serrated polyps in an average risk colorectal cancer screening cohort. Am J Gastroenterol 2010;105:2656-2664.

13. Kahi CJ, Hewett DG, Norton DL, Eckert GJ, Rex DK. Prevalence and variable detection of proximal colon serrated polyps during screening colonoscopy. Clin Gastroenterol Hepatol 2011;9:42-46.

14. de Wijkerslooth TR, Stoop EM, Bossuyt PM, et al. Differences in proximal serrated polyp detection among endoscopists are associated with variability in withdrawal time. Gastrointest Endosc 2013;77:617-623.

15. Butterly L, Robinson CM, Anderson JC, et al. Serrated and adenomatous polyp detection increases with longer withdrawal time: results from the New Hampshire Colonoscopy Registry. Am J Gastroenterol 2014;109:417-426.

16. Pohl H, Srivastava A, Bensen SP, et al. Incomplete polyp resection during colonoscopy-results of the complete adenoma resection (CARE) study. Gastroenterology 2013;144:74-80.e1

17. Pellise M, Burgess NG, Tutticci N, et al. Endoscopic mucosal resection for large serrated lesions in comparison with adenomas: a prospective multicentre study of 2000 lesions. Gut 2017;66:644-653.

18. Rex KD, Vemulapalli KC, Rex DK. Recurrence rates after EMR of large sessile serrated polyps. Gastrointest Endosc 2015;82:538-541.

19. Gao Q, Tsoi KK, Hirai HW, et al. Serrated polyps and the risk of synchronous colorectal advanced neoplasia: a systematic review and meta-analysis. Am J Gastroenterol 2015;110:501-509; quiz 510.

20. IJspeert JEG, Bevan R, Senore C, et al. Detection rate of serrated polyps and serrated polyposis syndrome in colorectal cancer screening cohorts: a European overview. Gut 2017;66:1225-1232.

21. Rivero-Sanchez L, Lopez-Ceron M, Carballal S, et al. Reassessment colonoscopy to diagnose serrated polyposis syndrome in a colorectal cancer screening population. Endoscopy 2017;49:44-53.

22. Hazewinkel Y, Tytgat KM, van Eeden S, et al. Incidence of colonic neoplasia in patients with serrated polyposis syndrome who undergo annual endoscopic surveillance. Gastroenterology 2014;147:88-95.

23. Carballal S, Rodríguez-Alcalde D, Moreira L, et al. Colorectal cancer risk factors in patients with serrated polyposis syndrome: a large multicentre study. Gut 2016;65:1829-1837.

24. Holme Ø, Bretthauer M, Eide TJ, et al. Long-term risk of colorectal cancer in individuals with serrated polyps. Gut 2015;64:929-936.

25. Erichsen R, Baron JA, Hamilton-Dutoit SJ, et al. Increased risk of colorectal cancer development among patients with serrated polyps. Gastroenterology 2016;150:895-902.e5.

26. Anderson JC, Butterly LF, Robinson CM, Weiss JE, Amos C, Srivastava A. Risk of metachronous high-risk adenomas and large serrated polyps in individuals with serrated polyps on index colonoscopy: data from the New Hampshire Colonoscopy Registry. Gastroenterology 2018;154:117127.e2. 